\title{
Non-anthocyanin polyphenols in healthy and Flavescence dorée infected Barbera and Nebbiolo leaves
}

\author{
Alessandra Ferrandino ${ }^{1}$, Chiara Pagliarani ${ }^{1,2}$, Olga Kedrina-Okutan ${ }^{1}$, Sara Icardi $^{1}$, Marco Bove ${ }^{1}$, Claudio \\ Lovisolo $^{1}$, Vittorino Novello ${ }^{1}$ and Andrea Schubert $^{1}$ \\ ${ }^{1}$ DISAFA, Dipartimento Scienze Agrarie, Forestali, Alimentari - University of Turin - Largo Braccini 2, 10095 Grugliasco \\ (TO), Italy \\ ${ }^{2}$ at present IPSP-CNR, Strada delle Cacce, 73, 10135 Torino, Italy.
}

\begin{abstract}
The first serious outbreak of Flavescence dorée (FD) in Piedmont (North-West Italy) dates back to 1998 in the Tortona area (Alessandria province). FD is a serious quarantine-worthy disease transmitted by the leafhopper Scaphoideus titanus Ball. Different Vitis vinifera L. biotypes react differently to the phytoplasma, in particular as to the accumulation of polyphenols in leaves. In this experimentation, we observed and described concentration and accumulation of the main classes of polyphenols in entire leaves and in leaf blades and veins of two varieties, Nebbiolo and Barbera, displaying different levels of susceptibility to FD. Their well-known different reactions could be related, at least partially, to leaf polyphenols, both as to concentrations and profiles. Nebbiolo displayed some specific traits: i) the higher percentage of incidence over totals of individual molecules known to be powerful antioxidants (caftaric acid over coutaric acid; quercetin glycosides over other flavonols); ii) the higher concentration of flavanols in veins with respect to Barbera and their wider profile (astilbin and a taxifolin-glycoside, this last accumulating exclusively in Nebbiolo).
\end{abstract}

\section{Introduction}

The first important outbreak of Flavescence dorée (FD) in Piedmont (North-West Italy) occurred in 1998; since that year FD has become a serious problem for Piedmont's regional viticulture, which has imposed compulsory sprays against the vector, the leafhopper Scaphoideus titanus Ball. The phytoplasma (FDp) inhabits the phloem, so in leaf veins the FD concentration is significantly higher than in blades (Christensen et al., 2004). In a previous field-experiment (Margaria et al., 2014), the effect of FD on leaf polyphenol accumulation and related gene expression in healthy, infected and recovered Barbera and Nebbiolo plants was described. The activation of the phenylpropanoid pathway following FD infection was evident in both varieties, and in Barbera the chalconesynthase 3 encoding gene (CHS3) was up to 20 -fold upregulated than in Nebbiolo; also genes encoding hydroxylases, UFGT, VvMYBA1 and LAR were upregulated in infected Barbera leaves with respect to Nebbiolo. This resulted in a significantly higher anthocyanin, flavonol and proanthocyanidin content, particularly at the end of the season (Margaria et al., 2014). One limit of that experiment was that the test vineyards were located in two different areas (there were no alternatives as this was the exclusive situation where two/three year-recovered plants were available). The second was that polyphenols were spectrophotometrically detected (except for flavonols), so we did not achieve any information about polyphenolic profiles. In the present work, we assessed the polyphenolic content and profile evolution in FDinfected Barbera and Nebbiolo leaves cultivated in controlled conditions over two consecutive seasons. In the second season, leaf blades and veins were analysed separately.

\section{Materials and Methods}

In 2014, one-year old Vitis vinifera vines of cv Barbera and Nebbiolo (kindly provided by the Roero Viti nursery, Montà d'Alba, CN) were grown in $60 \mathrm{~L}$ pots (filled with $50 \%$ of topsoil TS - Turco Silvestro, Albenga, SV, Italy, - $25 \%$ of clay and $25 \%$ of sand) in two separate greenhouses covered with white thick insect nets (Fig. 1).

\footnotetext{
Corresponding author: alessandra.ferrandino@unito.it
} 


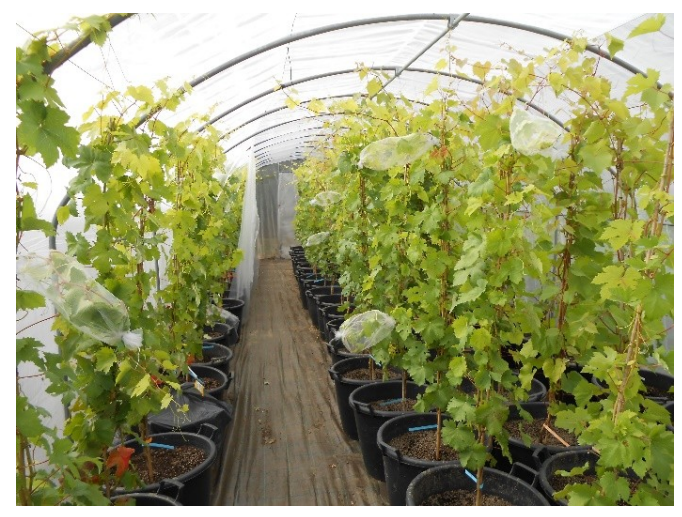

Fig. 1. Tunnel greenhouse hosting the trial.

Half of the vines were inoculated with FDp, strain C (the most prevalent in Piedmont), using infected Scaphoideus titanus Ball; the second half was used as healthy control. Vector insects were obtained in laboratory (detailed in Miliordos et al., 2017). In 2015 and 2016, all plants were screened for FDp presence/absence once a month from May to October by molecular diagnosis assay: genomic DNA was extracted accordingly to Marzachì et al., 1999 and the detection kit Flavescence dorée and Bois noir Tryplex real-time PCR (IPADLAB, Lodi) was used to detect the phytoplasma. At each sampling date, five leaves (collected from the basal, intermediate and apical part of the fruit-cane) were cut into pieces and extracted in a pH 3.9 buffer containing $40 \%$ ethanol. In 2016, leaf veins and blades were analysed separately. All analyses were performed in triplicates. Leaf flavonols, hydroxycinnamates (HCTAs) and monomeric proanthocyanidins were analysed by HPLC/DAD following well-established methods in our lab (Di Stefano and Cravero, 1992; Ferrandino and Guidoni, 2010). Individual molecule identification was performed by injection of the corresponding pure standard. Leaftissue specific molecules were identified by comparison with a HPLC/ESI/MS/MS peak identification of leaf polyphenols performed concomitantly (Kedrina-Okutan et al., submitted) and then further compared with the standards. Total concentrations were expressed as $\mathrm{mg} / \mathrm{kg}$ of the prevalent molecule: quercetin 3-O-glucuronide for flavonols and (+)-catechin for monomeric proanthocyanidins (both acquired from Extrasynthèse, Genay, France); caftaric acid for hydroxycinnamates (Sigma-Aldrich S.r.l., Milan, Italy). Astilbin was identified and quantified prior the injection of pure molecules to build the relative calibration curve (SigmaAldrich S.r.l., Milan, Italy); taxifolin hexoside was quantified as equivalents of astilbin.

\section{Results}

\subsection{Hydroxycinnamates}

Healthy Barbera leaves displayed a higher HCTA concentration with respect to Nebbiolo. HCTA concentration in FD-infected Barbera leaves increased during the season both when considering the entire leaf (Fig. 2) or veins and blades separately (Fig. 3).
Oppositely, in Nebbiolo, the HCTA concentration decreased during the season considering the whole leaf, and weakly increased in FD- infected veins at the time of the last sampling (Fig. 3). Caftaric acid was the prevalent HCTA in both varieties: its percentage incidence was significantly higher in Nebbiolo than Barbera, both in blades and in veins (Tab. 1).

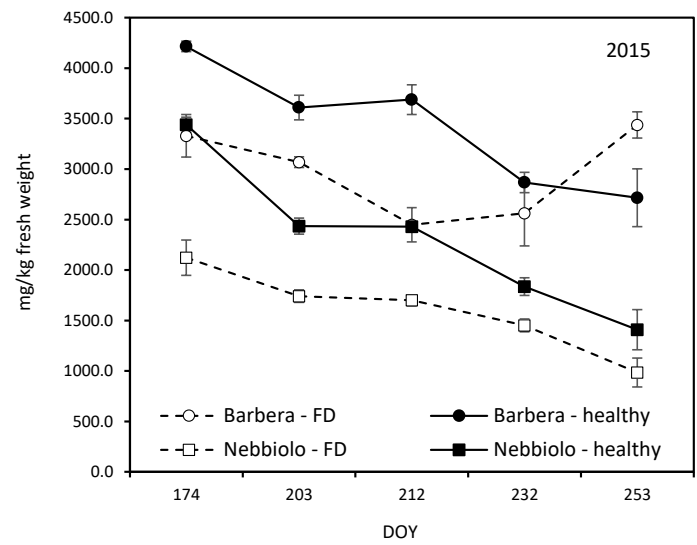

Fig. 2. Hydroxycinnamate concentration in healthy and FDinfected Barbera and Nebbiolo entire leaves in $2015(n=3 \pm$ s.e.).
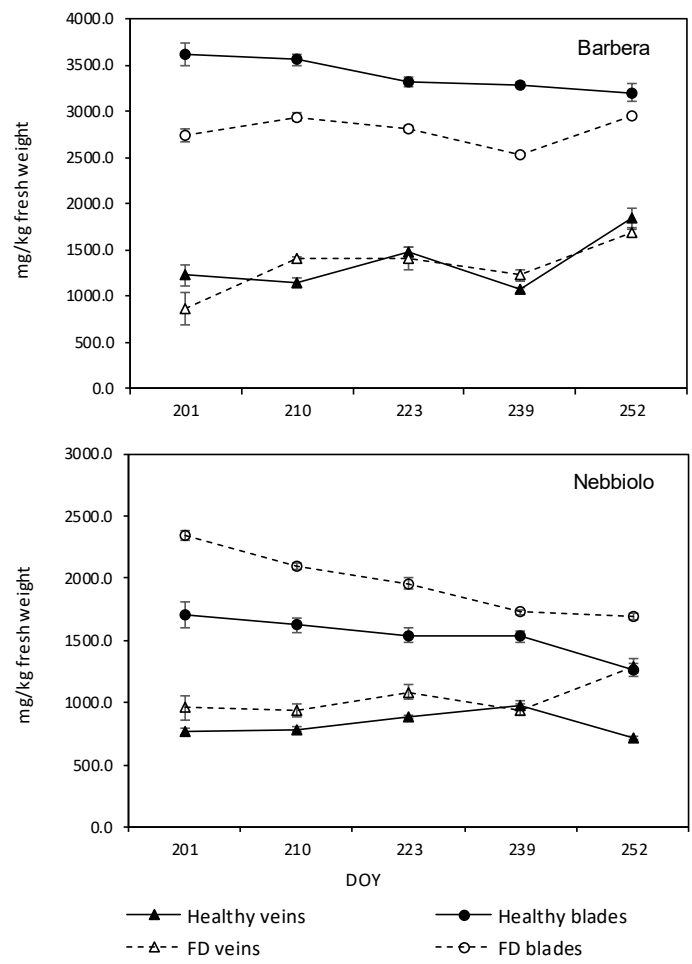

Fig. 3. Hydroxycinnamate concentration in healthy and FDinfected Barbera and Nebbiolo leaf veins and blades in 2016 (n $=3 \pm$ s.e.). 
Table 1. Caftaric acid (CA) and coutaric acid (CO) percentage incidence over total HCTA in healthy and FD-infected leaves (2015), veins and blades (2016). $\mathrm{S}=$ level of Significance by the Duncan's test at $*=\mathrm{P} \leq 0.05, * *=\mathrm{P} \leq 0.01$, between sanitary state (in columns) and varieties (in rows).

\begin{tabular}{|c|c|c|c|c|c|c|}
\hline & \multicolumn{2}{|c|}{ Barbera } & \multicolumn{2}{|c|}{ Nebbiolo } & \multicolumn{2}{|c|}{$\mathbf{S}$} \\
\hline & $\mathrm{CA}$ & $\mathrm{CO}$ & $\mathrm{CA}$ & $\mathrm{CO}$ & $\mathrm{CA}$ & $\mathrm{CO}$ \\
\hline \multicolumn{7}{|l|}{2015} \\
\hline $\begin{array}{l}\text { Healthy } \\
\text { leaves }\end{array}$ & $\begin{array}{c}82 . \\
7\end{array}$ & $\begin{array}{c}16 . \\
4\end{array}$ & 87.7 & $\begin{array}{c}11 . \\
8\end{array}$ & ** & $\mathrm{ns}$ \\
\hline $\begin{array}{l}\text { FD-inf } \\
\text { leaves }\end{array}$ & $\begin{array}{c}83 . \\
8\end{array}$ & $\begin{array}{c}15 . \\
5\end{array}$ & 88.8 & $\begin{array}{c}10 . \\
8\end{array}$ & $* *$ & ns \\
\hline $\mathbf{S}$ & ns & ns & ns & ns & & \\
\hline \multicolumn{7}{|l|}{2016} \\
\hline $\begin{array}{l}\text { Healthy } \\
\text { veins }\end{array}$ & $\begin{array}{c}83 . \\
9\end{array}$ & $\begin{array}{c}12 . \\
3\end{array}$ & 89.2 & 7.3 & $* *$ & $* *$ \\
\hline $\begin{array}{l}\text { Healthy } \\
\text { blades }\end{array}$ & $\begin{array}{c}82 . \\
5\end{array}$ & $\begin{array}{c}11 . \\
4\end{array}$ & 89.1 & 6.0 & $* *$ & $* *$ \\
\hline FD veins & $\begin{array}{c}85 . \\
3\end{array}$ & $\begin{array}{c}11 . \\
4\end{array}$ & 85.6 & 7.8 & ns & $*$ \\
\hline FD blades & $\begin{array}{c}81 . \\
0\end{array}$ & $\begin{array}{c}13 . \\
9\end{array}$ & 86.3 & 8.0 & $*$ & $* *$ \\
\hline S veins & ns & ns & $*$ & $\mathrm{~ns}$ & & \\
\hline $\mathrm{S}$ blades & ns & ns & * & ns & & \\
\hline
\end{tabular}

\subsection{Flavonols}

In 2015, when leaf tissue was analysed in toto (data not shown) flavonol accumulation was quite stable, regardless of the season and the plant sanitary status. Barbera leaves, as already noticed (Margaria et al., 2014; Kedrina-Okutan et al, submitted) showed a constitutive higher flavonol content with respect to Nebbiolo; the flavonol content was higher in blades than in veins, regardless the cultivar (Fig. 4). By analysing blades and veins separately, we found that in infected tissues in Nebbiolo, flavonol concentration was generally higher with respect to healthy ones, whereas the opposite happened in Barbera (Fig. 4). The profile of flavonols was influenced by the cultivar and the health status: indeed, the constitutive flavonol profile of Nebbiolo showed an average higher quercetin glycoside incidence than Barbera (Table 2), particularly at the first sampling; conversely, Barbera leaves had higher percentages of myricetin and kaempferol glycosides. Considering that many papers have long reported the higher antioxidant capacity of quercetin glycosides with respect to the other flavonol-glycosides, this aspect could help explain Nebbiolo's capacity to limit FDp development and spread.

Table 2. Quercetin glycosides (glucuronide + glucoside) average percentage incidence over the season in healthy and

FD-infected Barbera and Nebbiolo leaves. Averages were separated by the Duncan's test: $* \mathrm{P} \leq 0.05 ; * * \mathrm{P} \leq 0.01$;. Within

\begin{tabular}{|l|c|c|c|}
\hline & Barbera & Nebbiolo & significance \\
\hline Healthy veins & 81.8 & 89.1 & $* *$ \\
\hline Healthy blades & 83.6 & 89.8 & $*$ \\
\hline FD veins & 84.2 & 89.2 & $*$ \\
\hline FD blades & 85.5 & 90.2 & $*$ \\
\hline significance & $\mathrm{ns}$ & $\mathrm{ns}$ & \\
\hline
\end{tabular}

the same cultivar significance was tested for the sanitary status (healthy material vs FD-infected).
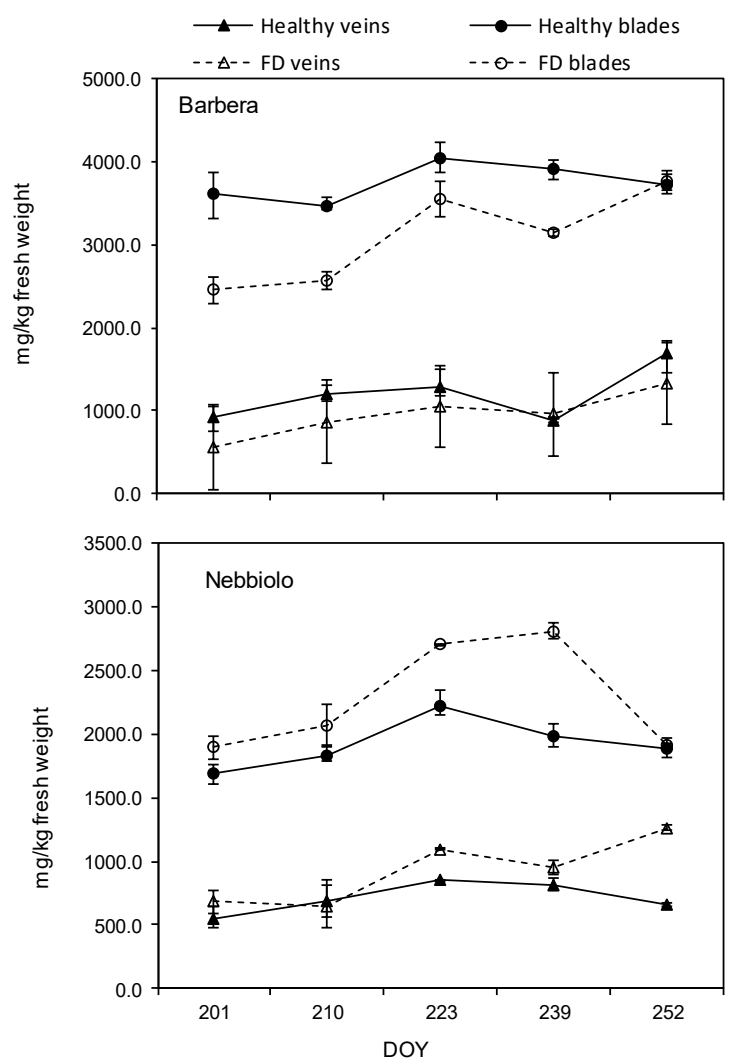

Fig. 4. Total flavonol evolution during the season in healthy and FD-infected Barbera and Nebbiolo leaf veins and blades (n $=3 \pm$ s.e. .

\subsection{Flavan 3-ols and flavanonols}

Detected monomeric flavan 3-ols included $(+)$-catechin, (-)-epicatechin, (-)-epigallocatechin and (-)-epicatechin gallate. Dimeric proanthocyanidin B1 was identified as well and, considering its different chromatographic response in the conditions adopted in this work (the calibration curve slope was one order of magnitude higher respect to that of monomeric forms (in line with Treutter et al., 1994), we deem it necessary to perform further identification and quantification trials to define absolute concentration of this molecule in response to FDp infection. However, comparing the two varieties (using the same calibration curves), the percentage incidence of B1 in Nebbiolo was higher with respect to Barbera (data not shown). In both varieties, the health status influenced the concentration of flavan 3-ols, being higher in FDp-infected tissues (Fig. 5). Even though the concentration of flavan 3-ols was generally higher in Barbera respect to Nebbiolo, FD-infected Nebbiolo blades and veins dramatically increased their concentration with respect to healthy ones (Fig. 6), which did not happen in Barbera.

Nebbiolo leaves also showed to be different according to total flavanonol concentration and profiles. Flavanonols were mostly accumulated in veins and in larger amounts in Nebbiolo compared to Barbera (Fig. 7). Flavanonol 
concentration increase in response to FD infection was evident in Nebbiolo but not in Barbera (Fig. 7). Furthermore, Nebbiolo displayed the widest profile variation accumulating astilbin (dihydroquercetinrhamnoside) and taxifolin (a dihydroquercetin-hexoside, in average seasonal proportions of $56-44 \%$ in healthy veins and $63-37 \%$ in FD-infected veins, respectively) whereas Barbera exclusively accumulated astilbin.

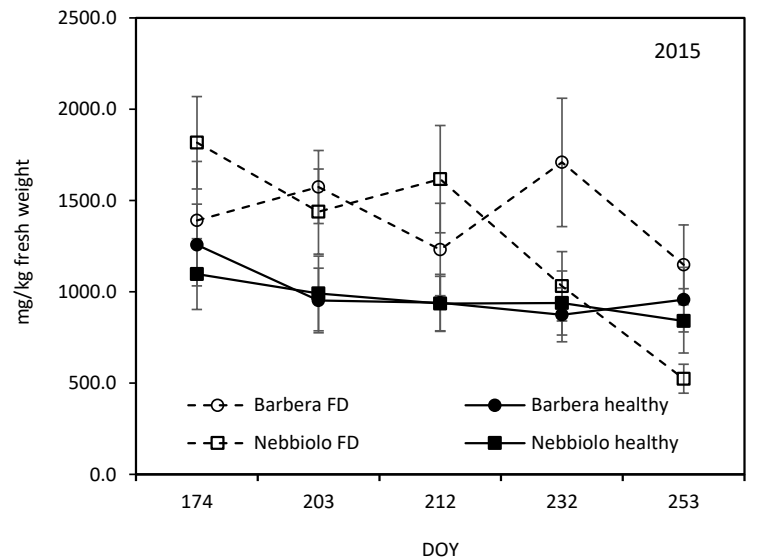

Fig. 5. Flavan 3-ols concentration in healthy and FD-infected Barbera and Nebbiolo entire leaves in $2015(n=3 \pm$ s.e. $)$.

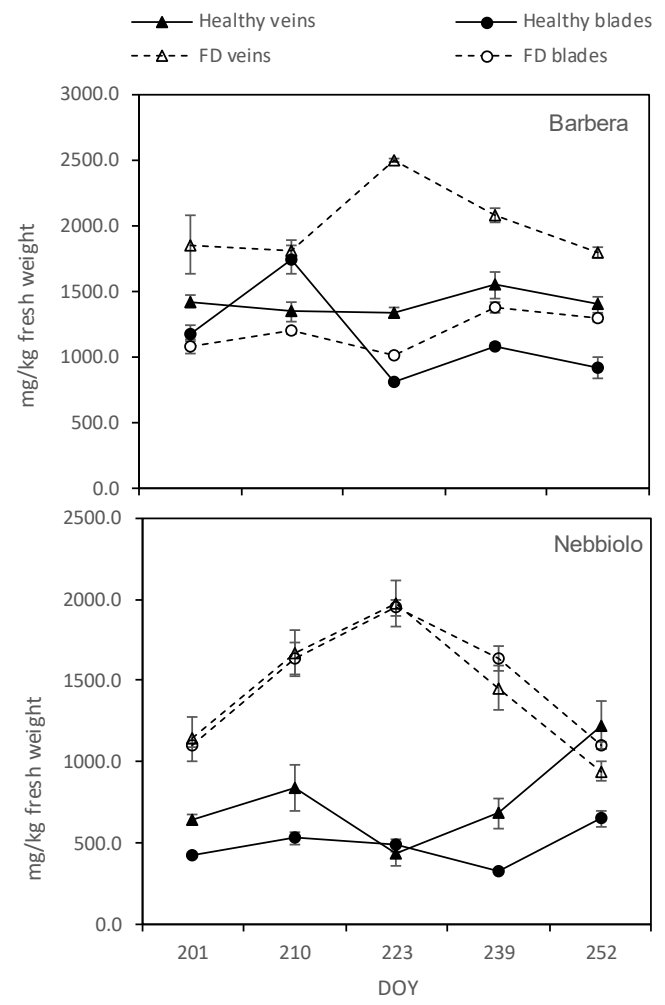

Fig. 6. Flavan 3-ols evolution during the season in healthy and FD-infected Barbera and Nebbiolo leaf veins and blades in $2016(n=3 \pm$ s.e. $)$.

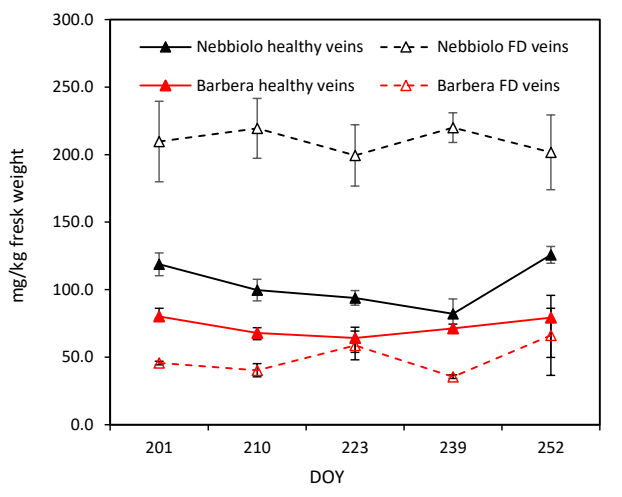

Fig. 7. Flavanonol evolution during the season in healthy and FD-infected Barbera and Nebbiolo leaf veins $(n=3 \pm$ s.e. $)$.

\section{Discussion}

HCTAs are generally considered as early-appearing intermediate products of the phenylpropanoid pathway; they are aspecific and their reduction in concentration can be ascribed to the biosynthesis of both flavonoids and stilbenes; a recent paper demonstrated that external distribution of caffeic acid increased the accumulation of stilbenes in Vitis amurensis (Tyunin et al., 2018). HCTAs are the most abundant non-flavonoid polyphenols in fruit juice; in grape berries this is due to the fact that they also accumulate in pulp rather than in skins. For this, their high concentration can counterbalance their lower antioxidant capacity respect to flavonoid polyphenols (anthocyanins and flavonols, in particular). In grape berries p-coumaric acid, the substrate of the formation of 4-coumaroyl- CoA, and cinnamic acid esterify with tartaric acid, forming coutaric acid and caftaric acid, a possible reservoir of the two cinnamic acids. Coumaric acid and caffeic acid, even simply differing for the level of hydroxylation, being coumaric acid mono-hydroxylated and caffeic acid di-hydroxylated, display dramatically different antioxidant capacities ( 0.6 vs 54 micromoles per L of $\mathrm{Cu}^{2+}$ oxidation according to Cartron et al., 2001). The higher percentage incidence of the caffeic acid ester in Nebbiolo leaves with respect to Barbera could represent a reservoir of this antioxidant, probably able to hinder specific pathogen proliferation into the vegetal tissue.

The higher antioxidant capacity of Nebbiolo leaves could also be related to the higher percentage incidence of quercetin derivatives, rather than other flavonol forms, being known that quercetins are among the highest antioxidant compounds known until now.

Additionally, Nebbiolo leaf extracts differed with respect to Barbera in other parameters: the higher percentage incidence of the dimeric proanthocyanidin B1 over the other monomeric flavan 3-ols, the highest concentration of flavanonols and their wider profile. It is noteworthy that flavanonols almost exclusively accumulated in veins, which make them particularly interesting in the study of the relation with phloem-located pathogens like FD. 


\section{Conclusions}

The well known different reaction of Nebbiolo to FD compared to Barbera could be related, at least partially, to leaf polyphenols, both as to their concentrations and profiles. Further studies related to other varieties with intermediate level of susceptibility to FD are under way to try to elucidate to what extent the phenylpropanoid pathway could be involved in the FD response of Vitis vinifera varieties.

\section{References}

1. A. Ferrandino, S. Guidoni, Eur. Food Res. Technol. 230, 417-427 (2010).

2. A.P. Tyunin, N. N. Nityagovsky, V. P. Grigorchuk, K. V. Kiselev, Biotech. Applied Biochem. 65, 150-155 (2018)

3. C. Marzachì, A. Alma, M. d'Aquilio, G. Minuto, G. Boccardo, J Plant Pathol. 81, 181187 (1999)

4. D. E. Miliordos, L. Galetto, E. Ferrari, M. Pegoraro, C. Marzachì, D. Bosco. Pest Manag. Sci. 73 (3), 534-540 (2017)

5. D. Treutter, Santos-Buelga C., Gutmann M., Kolodziej, J Chrom. A. 667 (1-2), 290-297 (1994)

6. E. Cartron, M.A. Carbonneau, G. Fouret, B. Descomps, C. L. Léger, J Nat. Prod. 64, 480-6 (2001)

7. N. M. Christensen, M. Nicolaisen, M. Hansen, A. Schulz, MPMI 17, 1175-1184 (2004)

8. O. Kedrina-Okutan, V. Novello, T. Hoffmann, J. Hardersdorfer, A. Occhipinti, W. Schwab, A. Ferrandino. Constitutive polyphenols in blades and veins of grapevine (Vitis vinifera L.) healthy leaves. Submitted.

9. P. Margaria, A. Ferrandino, P. Caciagli, O. Kedrina, A. Schubert, S. Palmano, Plant Cell Envir, 37(9), 2183-200 (2014).

10. R. Di Stefano, M.C. Cravero, M. C., Sci. des Aliment 12(1), 139-144 (1992). 